\title{
1 Over-expression of adaptor related protein complex 2 beta 1 subunit in human endometrial cancer.
}

Keywords: endometrial cancer, gynecologic cancers, endometrium, AP2B1, adaptor related protein complex 2 beta 1 subunit, systems biology of endometrial cancer, targeted therapeutics in endometrial cancer.

Shahan Mamoor, $\mathrm{MS}^{1}$

${ }^{1}$ shahanmamoor@gmail.com

East Islip, NY USA

Gynecologic cancers including cancers of the endometrium are a clinical problem ${ }^{1-4}$. We mined published microarray data ${ }^{5,6}$ to discover genes associated with endometrial cancers by comparing transcriptomes of the normal and hyperplastic endometrium to endometrial tumors from humans. We identified adaptor related protein complex 2 beta 1 subunit, encoded by AP2B1, as among the most differentially expressed genes, transcriptome-wide, in cancers of the endometrium. AP2B1 was expressed at significantly higher levels in endometrial tumor tissues as compared to the endometrium. Importantly, in human endometrial cancer, primary tumor expression of AP2B1 was correlated with overall survival in white patients with low mutational burden. AP2B1 may be a molecule of interest in understanding the etiology or progression of human endometrial cancer. 
Endometrial cancer is the most common gynecologic cancer in the developed world ${ }^{1}$. Over the last three decades, the incidence of endometrial cancer has increased $21 \%{ }^{4}$ and the death

\section{$\underline{\text { Methods }}$}

We utilized datasets GSE63678 5 and GSE106191 ${ }^{6}$ for this global differential gene datasets $^{5,6}$ to determine in an unbiased fashion and at the systems-level genes most differentially expressed in endometrial tumors. We report here the differential and increased expression of the adaptor related protein complex 2 beta 1 subunit (AP2B1) in human endometrial cancer. expression analysis of human endometrial cancer in conjunction with GEO2R. GSE63678 was generated using Affymetrix Human Genome U133A 2.0 Array technology with $n=5$ control endometrial tissues (including $n=4$ uterine myomas and $n=1$ benign cyst) and $n=7$ endometrial cancers (including $n=2$ endometrial adenocarcinomas, $n=3$ mixed endometrioid adenocarcinomas, and $n=2$ adenocarcinomas with squamous differentiation); analysis was performed using platform GPL571. GSE106191 was generated using Affymetrix Human Genome U133 Plus 2.0 Array technology with $n=33$ hyperplastic endometrial tissues and $n=28$ endometrial cancer tissues; analysis was performed using platform GPL570. The Benjamini and Hochberg method of $p$-value adjustment was used for ranking of differential expression but raw $p$-values were used to assess statistical significance of global differential expression.

Log-transformation of data was auto-detected, and the NCBI generated category of platform annotation was used. A statistical test was performed to evaluate whether AP2B1 gene expression was significantly different between control endometrial tissue and endometrial tumor tissue in humans using a two-tailed t-test. For Kaplan-Meier survival analysis, we used the Kaplan-Meier plotter tool ${ }^{7}$ for correlation of AP2B1 mRNA expression levels with overall survival in $n=543$ endometrial cancer patients.

\section{$\underline{\text { Results }}$}

We harnessed the power of blind comparative transcriptome analysis using published microarray data ${ }^{5,6}$ to discover in an unbiased fashion genes associated with endometrial cancer in humans.

\section{AP2B1 is differentially expressed in endometrial cancer.}

We identified adaptor related protein complex 2 beta 1 subunit, encoded by AP2B1, as among the genes most differentially expressed in cancers of the endometrium when compared to benign endometrial tissues (Chart 1). When sorting each of the genes expressed in endometrial tumor tissue based on significance of change in expression as compared to benign endometrial tissue, AP2B1 ranked 164 out of 22273 transcripts, equating to $99.3 \%$ differential expression (Chart 1). Differential expression of AP2B1 in human endometrial cancers was statistically significant (Chart $1 ; p=1.88 \mathrm{E}-04)$. 
We queried a second microarray data to validate differential expression of AP2B1 in endometrial cancer. Here, we observed differential expression of AP2B1 when comparing endometrial tumor tissue to hyperplastic endometrial tissue (Chart 2). When sorting each of the genes expressed in endometrial tumor tissue based on significance of change in expression as compared to hyperplastic endometrial tissue, AP2B1 ranked 4982 out of 54675 transcripts, equating to $90.9 \%$ differential expression (Chart 2). Differential expression of AP2B1 in human endometrial cancers was statistically significant (Chart $2 ; p=2.66 \mathrm{E}-02$ ).

\section{AP2B1 is expressed at significantly higher levels in endometrial cancers as compared to benign endometrial tissue.}

We obtained exact mRNA expression levels for AP2B1 in endometrial tumor tissues and from benign endometrial tissue to evaluate direction and statistical significance of change in expression of AP2B1 in human endometrial cancer. AP2B1 was expressed at higher levels in endometrial tissue as compared to normal endometrial tissue, and this difference was statistically significant (Figure 1; $p=0.0013$ ). We calculated a mean fold change of 1.12 in AP2B1 mRNA levels in human endometrial cancer, as AP2B1 was expressed at $8.01 \pm 0.36$ arbitrary units (A.U.) in control endometrial tissue but at $8.97 \pm 0.28$ A.U. in endometrial tumor tissue.

\section{AP2B1 expression is correlated with patient survival outcomes in endometrial cancer.}

We performed Kaplan-Meier survival analysis to evaluate correlation between AP2B1 primary tumor expression and survival outcomes in 543 patients with endometrial cancer. We observed a correlation between primary tumor expression of AP2B1 and overall survival in patients with endometrial cancer, in white patients with low mutational burden, in the lower survival tertile (Figure 2). AP2B1 primary tumor mRNA levels were a negative prognostic indicator in white endometrial cancer patients with low mutational burden. White patients with low mutational burden whose primary tumors expressed low levels of AP2B1 possessed, on average, markedly greater median OS as compared to white patients with low mutational burden whose tumors expressed high levels of AP2B1 (Figure 2). This difference in OS based on AP2B1 tumor expression in white patients with endometrial cancer with low mutational burden was statistically significant (Figure 2, Chart 3; logrank $p$-value: 0.041; hazard ratio: 2.31 (1.01-5.3)). AP2B1 primary endometrial tumor expression was not correlated with overall survival in white patients with high mutational burden (Figure 2, Chart 3; logrank $p$-value: 0.84; hazard ratio: 1.09 (0.48-2.47)), nor in black patients with high ((Figure 2, Chart 3; logrank p-value: 0.38; hazard ratio: $2.55(0.3-21.83)$ ) or low mutational burden (Figure 2, Chart 3; logrank $p$-value: 0.76 ; hazard ratio: $0.84(0.28-2.54))$.

Thus, by mining published microarray data ${ }^{5,6}$ in an unbiased and systematic fashion, we identified adaptor related protein complex 2 beta 1 subunit, encoded by AP2B1, as among the genes whose expression was most different, transcriptome-wide, in the endometrial tumor tissue 


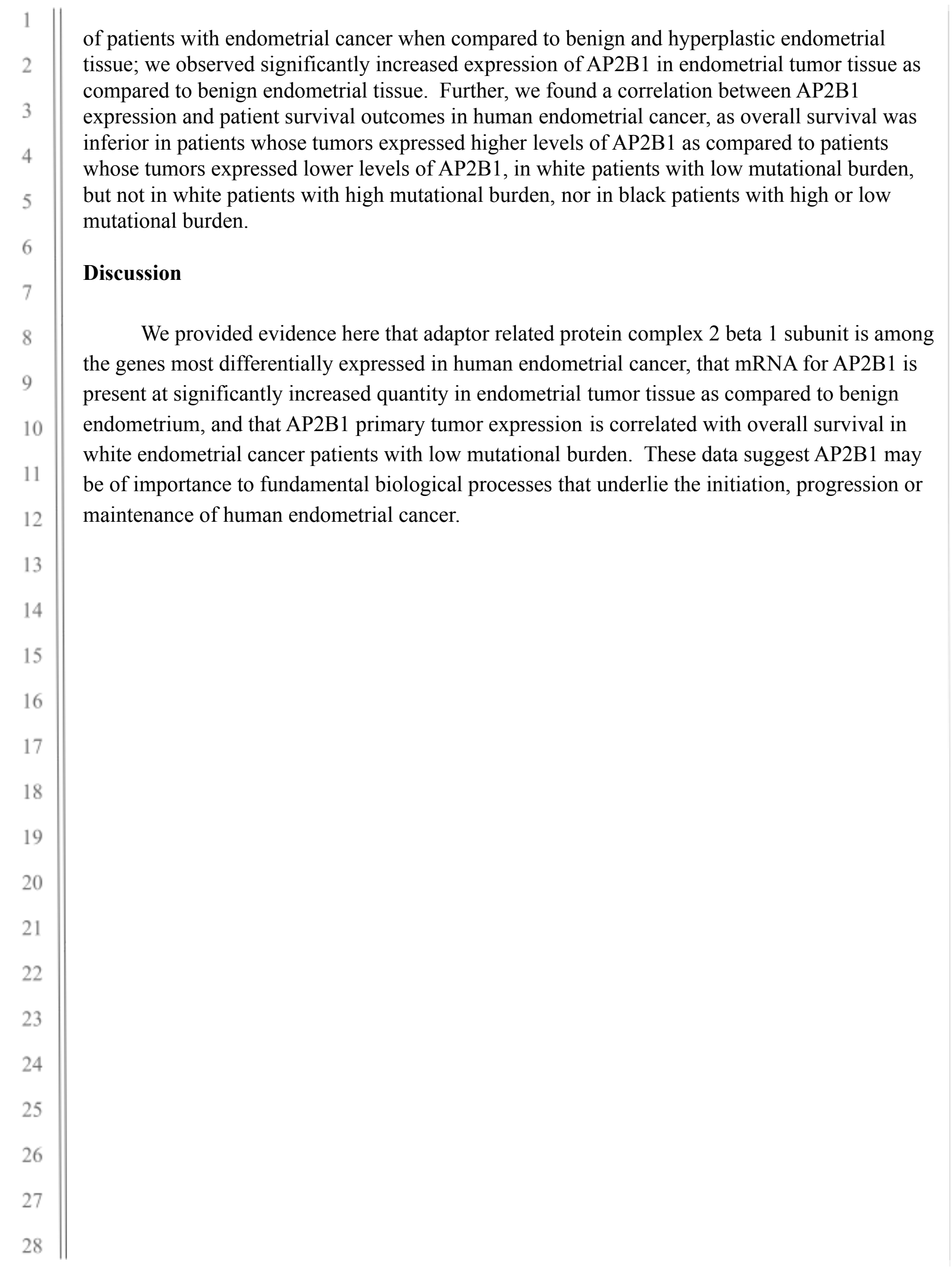




\section{References}

1. Amant, F., Moerman, P., Neven, P., Timmerman, D., Van Limbergen, E. and Vergote, I., 2005. Endometrial cancer. The Lancet, 366(9484), pp.491-505.

2. Sorosky, J.I., 2008. Endometrial cancer. Obstetrics \& Gynecology, 111(2), pp.436-447.

3. Morice, P., Leary, A., Creutzberg, C., Abu-Rustum, N. and Darai, E., 2016. Endometrial cancer. The Lancet, 387(10023), pp.1094-1108.

4. Sorosky, J.I., 2012. Endometrial cancer. Obstetrics \& Gynecology, 120(2 Part 1), pp.383-397.

5. Pappa, K.I., Polyzos, A., Jacob-Hirsch, J., Amariglio, N., Vlachos, G.D., Loutradis, D. and Anagnou, N.P., 2015. Profiling of discrete gynecological cancers reveals novel transcriptional modules and common features shared by other cancer types and embryonic stem cells. PLoS One, 10(11), p.e0142229.

6. Sugiyama, Y., Gotoh, O., Fukui, N., Tanaka, N., Hasumi, K., Takazawa, Y., Noda, T. and Mori, S., 2020. Two distinct tumorigenic processes in endometrial endometrioid adenocarcinoma. The American journal of pathology, 190(1), pp.234-251.

2 7. Nagy, A., Munkacsy, G. and Gyorffy, B., 2020. Pancancer survival analysis of cancer hallmark genes. bioRxiv. 
Rank: 164

Probe ID: 200615_s_at

p-value: $1.88 \mathrm{E}-04$

3 t: -5.2726343

$4 \quad$ B: 1.05171

Gene: AP2B1

5 Gene name: adaptor related protein complex 2 beta 1 subunit

Chart 1: AP2B1 is differentially expressed in endometrial cancer when comparing primary endometrial tumors to benign endometrial tissue.

The rank of global differential expression, probe/transcript ID, the $p$-value with respect to

9 differential expression transcriptome-wide, $t$, a moderated $t$-statistic, $\mathrm{B}$, the log-odds of differential expression between the groups compared, the gene and gene name are listed in this chart. 


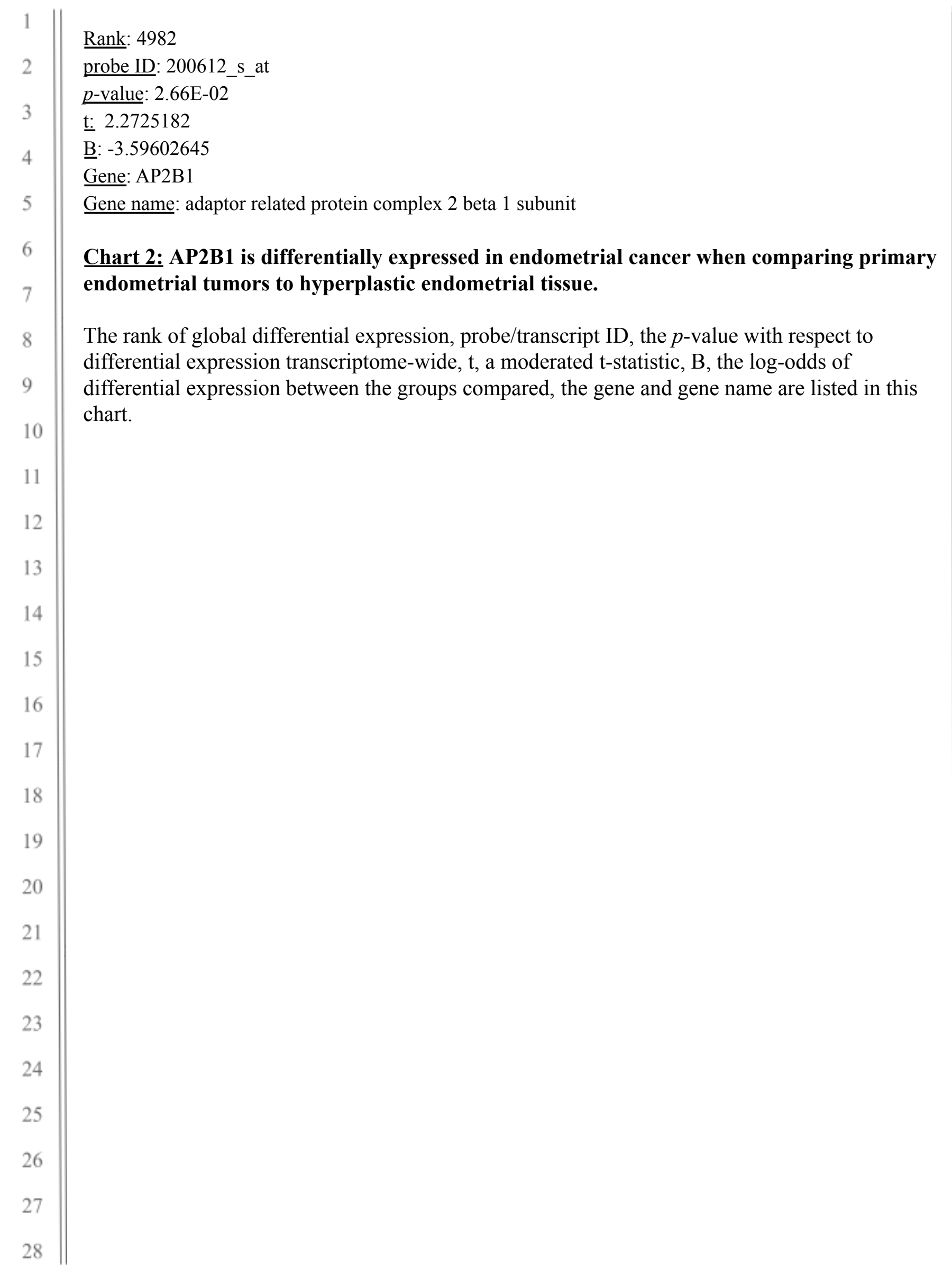




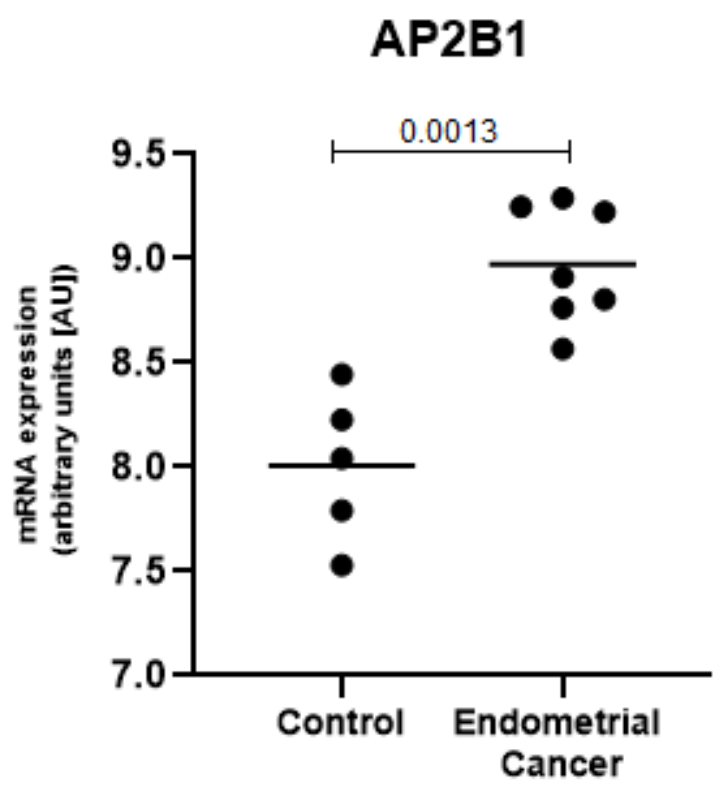

Figure 1: AP2B1 is expressed at significantly higher levels in the endometrial tumors of patients with endometrial cancer when compared to benign endometrium.

The mRNA expression level of AP2B1 in benign endometrial tissue (left) and in primary tumors of the endometrium (right) is graphically depicted; the result of a statistical test evaluating significance of difference in AP2B1 expression between benign endometrial tissue and primary tumors of the endometrial tissue is $p=0.0013$. 


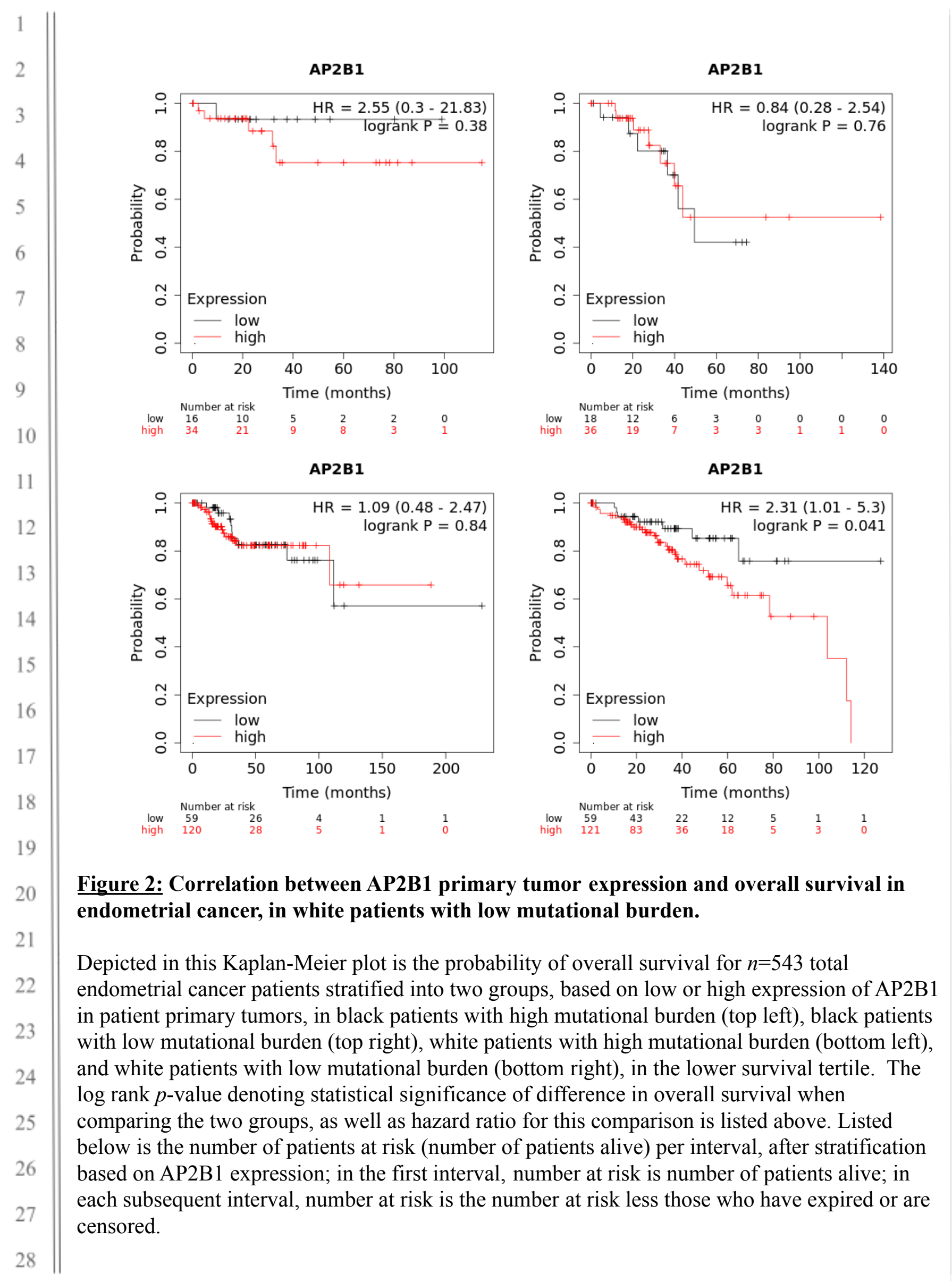

